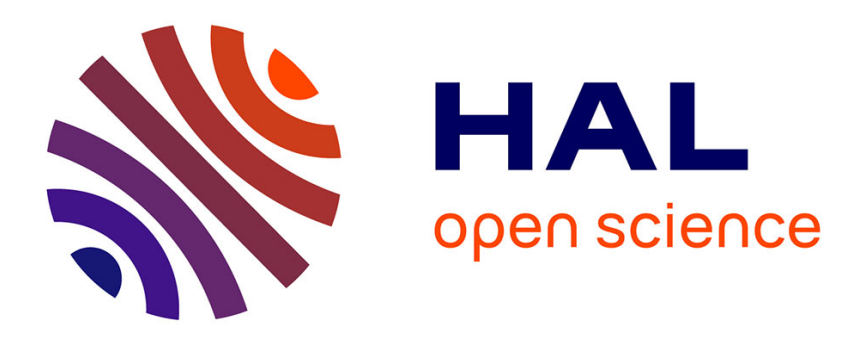

\title{
The budding yeast life cycle: More complex than anticipated?
}

Gilles Fischer, Gianni Liti, Bertrand Llorente

\section{To cite this version:}

Gilles Fischer, Gianni Liti, Bertrand Llorente. The budding yeast life cycle: More complex than anticipated?. Yeast, 2021, 38 (1), pp.5-11. 10.1002/yea.3533 . hal-03086108

\section{HAL Id: hal-03086108 \\ https://hal.science/hal-03086108}

Submitted on 4 Jan 2021

HAL is a multi-disciplinary open access archive for the deposit and dissemination of scientific research documents, whether they are published or not. The documents may come from teaching and research institutions in France or abroad, or from public or private research centers.
L'archive ouverte pluridisciplinaire HAL, est destinée au dépôt et à la diffusion de documents scientifiques de niveau recherche, publiés ou non, émanant des établissements d'enseignement et de recherche français ou étrangers, des laboratoires publics ou privés. 
The Budding Yeast Life Cycle: more complex than anticipated?

Gilles Fischer ${ }^{1}$, Gianni Liti $^{2}$ and Bertrand Llorente ${ }^{3}$

Affiliations:

${ }^{1}$ Sorbonne Université, CNRS, Institut de Biologie Paris-Seine, Laboratory of Computational and Quantitative Biology, Paris, France

${ }^{2}$ Université Côte d'Azur, CNRS, INSERM, IRCAN, Nice, France

${ }^{3}$ Cancer Research Center of Marseille, CNRS, Inserm, Institut Paoli-Calmettes, Aix-Marseille Université, Marseille, France 


\section{Abstract}

The budding yeast, Saccharomyces cerevisiae has served as a model for nearly a century to understand the principles of the eukaryotic life cycle. The canonical life cycle of $S$. cerevisiae comprises a regular alternation between haploid and diploid phases. Haploid gametes generated by sporulation are expected to quickly restore the diploid phase mainly through inbreeding via intra-tetrad mating or haploselfing, thereby promoting genome homozygotization. However, recent large population genomics data unveiled that heterozygosity and polyploidy are unexpectedly common. This raises the interesting paradox of a haplo-diplontic species being well-adapted to inbreeding but also able to maintain high levels of heterozygosity and polyploidy, thereby suggesting an unanticipated complexity of the yeast life cycle. Here, we propose that unprogrammed mating type switching, heterothallism, reduced spore formation and viability, cell-cell fusion and dioecy could play key and uncharted contributions to generate and maintain heterozygosity through polyploidization. 


\section{An intriguing paradox in the yeast life cycle}

A large-scale population genomic surveys in Saccharomyces cerevisiae recently unveiled the quantitative importance of heterozygous and polyploid isolates in both domesticated and wild populations [Peter et al. 2018]. In addition, there is a striking association between heterozygosity and polyploidy, all triploid and tetraploid isolates being highly heterozygous. Such genomic makeup suggests an unanticipated complexity of the yeast life cycle, which instead is expected to produce mostly homozygous diploids. Indeed, the classical budding yeast life cycle consists of an alternation between diploid and haploid phases where diploid cells proliferate mitotically under nutrient rich conditions and undergo meiosis when nutrients become limited to produce tetrads composed of four haploid spores. Upon germination, spores from opposite mating types mate and restore diploids therefore promoting homozygosity. Alternatively, haploid mother cells can switch their mating type allowing them to mate with their daughters to autodiploidize by haploselfing, again leading to homozygous diploids. While outcrossing resulting from inter-tetrad mating between different lineages can generate heterozygous diploids, numerous studies have shown that the two aforementioned inbreeding strategies through intra-tetrad mating and haploselfing, respectively, largely exceed outcrossing (see below).

There are two mutually non-exclusive possible causes for why heterozygosity and polyploidy are observed more frequently than expected: either they originate at unexpectedly high rates or they persist longer than assumed. Origin can be purely random or programmed. Persistence can result from chance or from selection. In this perspective, after reviewing the canonical budding yeast life cycle, we describe how recent population genomics data depart from the expectations and propose possible mechanisms leading to such complexity.

\section{The canonical Saccharomyces cerevisiae life cycle}

In yeasts, life cycles comprise intertwined phases of asexual clonal expansion, sexual reproduction and quiescence. The classical description of the budding yeast life cycle is haplo-diplobiontic. Haploid cells from opposite cellular types mate to form diploids, which proliferate mitotically under nutrient rich conditions. Diploids undergo meiosis under nutrient poor conditions and produce tetrads composed of four haploid spores enclosed within an ascus. Additionally, S. cerevisiae is homothallic i.e. haploid cells can change their mating type during vegetative growth by a programmed switching mechanism. This allows haploid mother cells to switch mating type after generating a daughter cell. Mother and daughter cell mating re-establishes the diploid state by haploselfing (Figure 1). The MAT locus determines the mating type of haploid cells and encodes either the a1 gene (MATa) or the $\alpha 1$ and $\alpha 2$ genes (MATa). Mating type switching in $S$. cerevisiae is one of the best understood programmed DNA recombination events. The site-specific endonuclease HO induces a DNA 3 
Double Strand Break (DSB) at MAT. This DSB is repaired by homologous recombination (HR) using as a template one of the two silent cassettes $H M L$ and HMR that carry the $\alpha$ and $a$ information, respectively. Switching occurs by a gene conversion event that replaces the preexisting mating type at the MAT locus by the opposite type coded at either $H M L$ or $H M R$ [Haber 1998]. Haploselfing is restricted to homothallic isolates. However, both homothallic and heterothallic strains can produce diploids by mating between two haploid cells resulting from the germination of spores of opposite mating types. The two spores can originate either from the same ascus or different asci (intra- or inter-tetrad mating, respectively, Figure 1). The different life cycle processes are likely quantitative traits and controlled by a large number of quantitative trait loci (QTLs). Linkage and GWAS analyses identified multiple sporulation QTLs [Gerke, Lorenz, and Cohen 2009; Chiara, Barré, Persson, Chioma, Irizar, Schacherer, Warringer, and Liti 2020]. Spore viability and mating efficiency are also likely complex whereas mating type switching efficiency might be largely explained by variations in a more limited number of loci comprising the $\mathrm{HO}$ gene, its target sequence and the recombination enhancer [Haber 2012]. In conclusion, the consensus view on the budding yeast life cycle mainly stems from decades of laboratory experiments but its natural complexity in the wild remains largely unknown [Liti 2015].

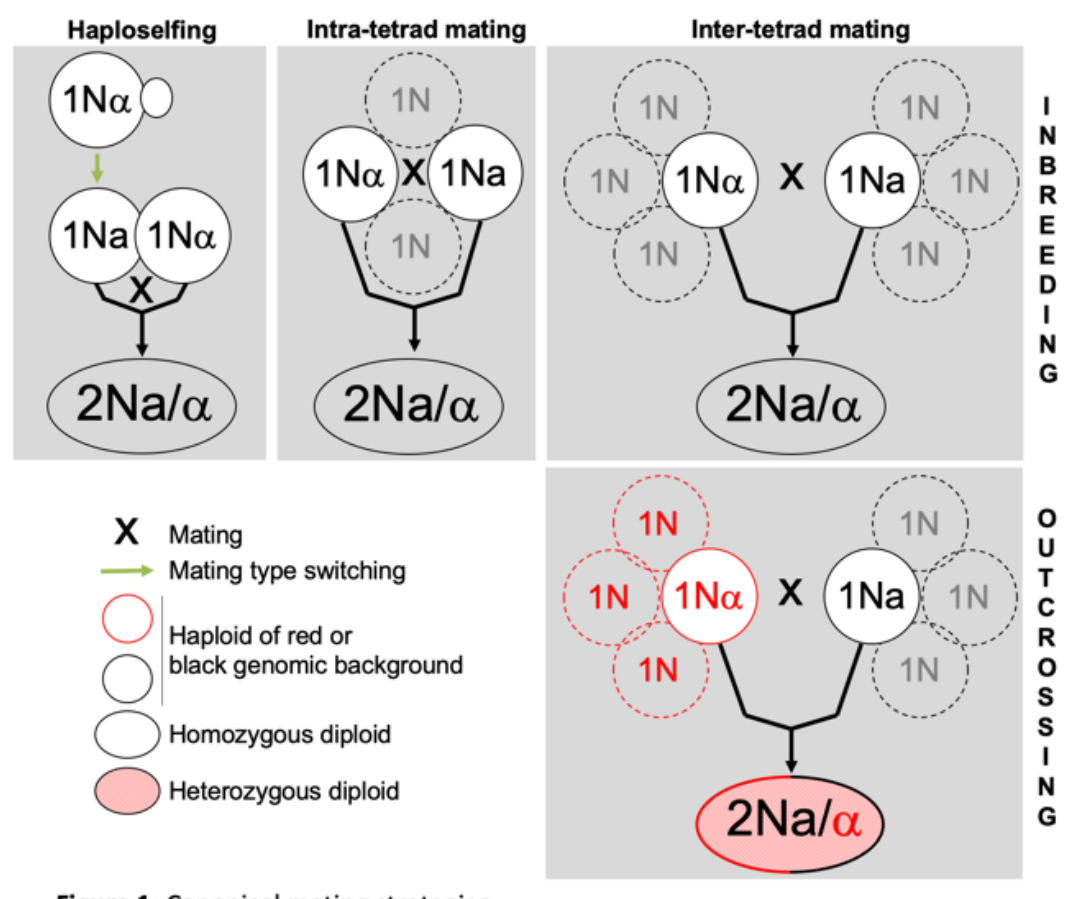

Figure 1: Canonical mating strategies

\section{Box 1: Life cycle characteristics in Saccharomyces cerevisiae}


Dioecy: Separation of sexes. A dioecious strain produces by meiosis spores of only one mating type either MATa or MATa but not both.

Haplo-diplobiontic: Alternations between haploid and diploid stages during the life cycle.

Haploselfing: Autodiploidization after a mitotic cell division where the haploid mother cell switches its mating type and mates with its daughter cell.

Heterothallism: An heterothallic haploid yeast cell has a stable mating type, can mate with an haploid of opposite mating type but cannot undergo mother-daughter mating by haploselfing.

Homothallism: An homothallic yeast strain can undergo mating-type switching and haploselfing.

Inbreeding: Crossing between two spores from the same tetrad or from two different tetrads from the same clonal lineage.

Outcrossing: Crossing between two spores from unrelated lineages.

Polyploid: A cell that has more than two complete sets of chromosomes.

\section{Prevalence of heterothallism}

Most laboratory experiments have conveniently used natural heterothallic isolates carrying four non-synonymous mutations in the $\mathrm{HO}$ gene including a leucine to histidine substitution within a zinc finger domain responsible for the loss of the endonuclease function [Meiron, Nahon, and Raveh 1995]. Indeed, heterothallic strains are unable to switch mating types and therefore can be propagated as stable haploids. Other mutations have been reported to reduce MAT switching efficiency by reducing $\mathrm{HO}$ cleavage efficiency through mutations in the $\mathrm{HO}$ gene or in its recognition site [Haber, Savage, Raposa, Weiffenbach, and Rowe 1980; Ray, White, and Haber 1991]. A field sampling campaign in Israel "Evolution Canyon" suggested that the proportion of naturally occurring heterothallic isolates might have been underappreciated [Ezov, Boger-Nadjar, Frenkel, Katsperovski, Kemeny, Nevo, Korol, and Kashi 2006]. Most of the spores derived from the 21 Israeli diploid strains developed as stable haploids, being unable to switch mating type. HO sequencing revealed many nonsense, missense and frameshift mutations, including all four non-synonymous substitutions that are present in laboratory strains [Ezov, Chang, Frenkel, Segrè, Bahalul, Murray, Leu, Korol, and Kashi 2010]. These findings raise questions on the quantitative occurrence of naturally heterothallic isolates and the limitation of the ability to undergo haploselfing at the population level. We tackled this question by examining the diversity of 
HO gene sequences across 1,011 genomes [Peter et al. 2018]. Protein sequence alignments reveal both dramatic gene length variation (with many truncated alleles) and numerous amino-acid substitutions (Figure 2). Both small and highly diverged alleles are likely inactive, suggesting that a large proportion of strains would be unable to switch mating-type and therefore be heterothallic.

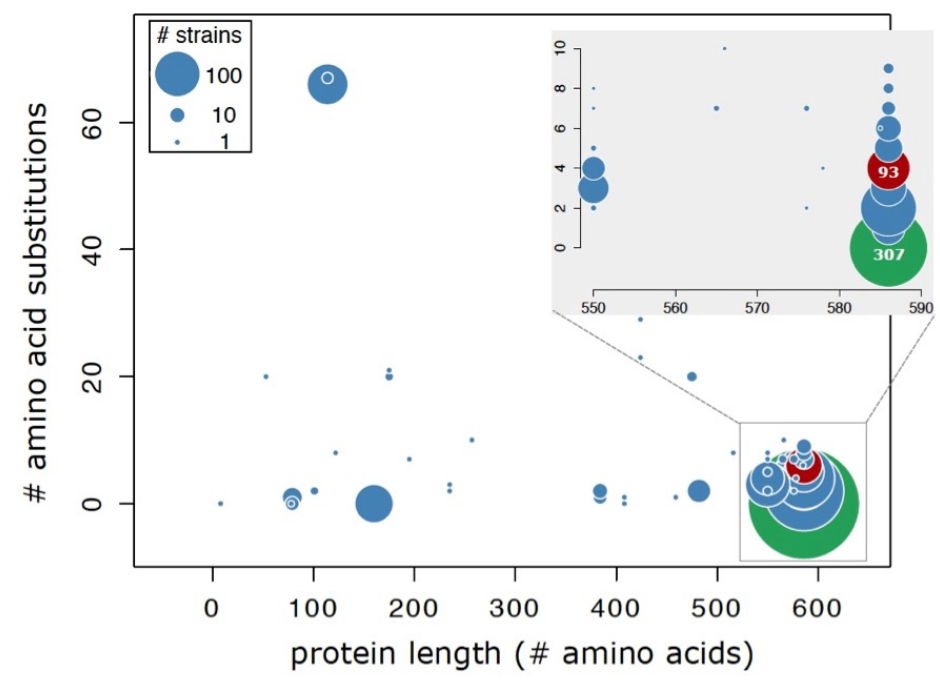

Figure 2: $\mathrm{HO}$ gene diversity across the 1,011 genomes. The circle diameter is proportional to the number of strains. The red circle comprise 93 strains and represents the inactive form of $\mathrm{HO}$ present in most laboratory strains. The green circle comprises 307 strains carrying the active HO sequence. The blue circles contain strains for which HO functionality is unknown. However, all truncated forms of the $\mathrm{HO}$ gene are likely heterothallic.

\section{Unexpected high level of heterozygosity}

The most comprehensive population genomic study, including 1,011 S. cerevisiae strains [Peter et al. 2018], revealed that $63 \%$ of the isolates analyzed were heterozygous with numerous strains exhibiting thousands of heterozygous polymorphisms (Figure 3). Such a high heterozygosity level is hardly compatible with a life cycle where inbreeding and mitotic $\mathrm{LOH}$ would dominate and rapidly lead to homozygosity. Various studies either based on population genomics data or on experimental systems have tried to estimate the relative contribution of the different mating strategies and their impact on heterozygosity, sometimes generating contradictory results. 


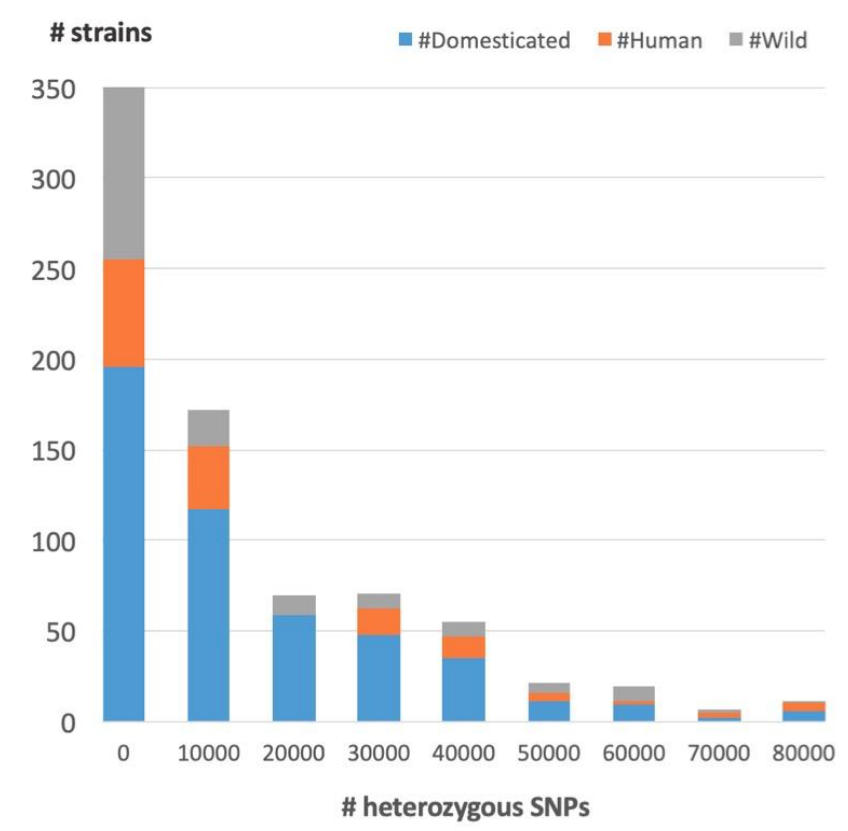

Figure 3: Distribution of the number of heterozygous SNPs in the 794 strains sequenced in their original ploidy and heterozygosity levels (Peter et al., Nature 2018) as a function of their ecological niche. "Domesticated" means strains associated with human activity (fermentation, bakery), "Human" means strains isolated from human body or human-related environment such as cultivated fruits and flowers, "Wild" means strains isolated from natural environments

These studies showed that inbreeding through intra-tetrad mating was the main mating mechanism (60 to $90 \%$ of the mating events) while haploselfing and inbreeding through intertetrad mating contributed similarly and accounted for the rest of the cases [Taxis, Keller, Kavagiou, Jensen, Colombelli, Bork, Stelzer, and Knop 2005; Ruderfer, Pratt, Seidel, and Kruglyak 2006; Murphy and Zeyl 2010; Nishant et al. 2010; McClure, Jacobs, Zyla, and Lew 2018]. Outcrossing events by inter-tetrad mating between different lineages was very rare, occurring once every $50,000-100,000$ mitotic divisions in S. cerevisiae and only in $1 \%$ of the mating events in wild S. paradoxus [Ruderfer, Pratt, Seidel, and Kruglyak 2006; Tsai, Bensasson, Burt, and Koufopanou 2008]. Consistently, the seminal Robert Mortimer's "Genome Renewal" hypothesis predicts that rare sexual cycles combined with haploselfing promote fixation of beneficial alleles and purging of deleterious mutations accumulated during long periods of vegetative growth [Mortimer, Romano, Suzzi, and Polsinelli 1994]. A single haploselfing event leads to complete homozygosity, except at the mating type locus. Inbreeding through both inter- and intra-tetrad mating is also expected to promote a rapid loss of heterozygosity, such that $82 \%$ and $70 \%$ of genome-wide heterozygosities would be lost after 3 meiotic generations, respectively [Knop 2006]. This theoretical scenario has been supported by laboratory experiments showing that the number of heterozygous SNPs rapidly declined with an increasing number of meiotic generations. For instance, after three and seven rounds of meiosis and inbreeding, $70 \%$ and $90 \%$ of the heterozygous SNPs were 
respectively lost [Dutta, Lin, Pankajam, Chakraborty, Bhat, Steinmetz, and Nishant 2017]. In addition, rare sexual cycles are separated by active phases of clonal expansion during which mitotic recombination can occur thereby promoting extensive loss-of-heterozygosity (LOH) regions [Peter et al. 2018; Llorente, Smith, and Symington 2008; Tattini, Tellini, Mozzachiodi, D’Angiolo, Loeillet, Nicolas, and Liti 2019]. Under such a scenario where inbreeding prevails during sexual cycles and $\mathrm{LOH}$ frequently occurs during mitotic cycles, it is expected that heterozygosity should be extremely low, with the generation of heterozygous diploids being restricted to rare outcrossing events between spores from diverged lineages (Figure 1).

However, other studies suggested that outcrossing in $S$. cerevisiae may be considerably more frequent than previously reported [Kelly, Shewmaker, Kryndushkin, and Wickner 2012] and promoted by specific conditions such as insect guts [Reuter, Bell, and Greig 2007; Goddard, Anfang, Tang, Gardner, and Jun 2010]. In addition, sporulation under carbon source limitation leads to a reduced number of spore per tetrad (down to single spore monads), thereby reducing or abolishing intra-tetrad mating possibilities [Taxis et al. 2005].

A possible scenario to explain this conundrum is that human activities would bring together diverged lineages and human-related processes such as baking or beverage fermentations would favor outcrossing [Magwene 2014]. Heterozygotes might be maintained because they are fitter in domesticated environments for traits related to brewing, baking or other biotechnological processes. Under such scenario, wild isolates are instead expected to be mainly homozygous. For instance, wild Chinese lineages are all homozygotes while domesticated strains comprise numerous heterozygotes [Duan, Han, Wang, Liu, Shi, Li, Zhang, and Bai 2018]. However, heterozygous Chinese strains that cluster with Baiju or Mantou domesticated lineages were isolated from wild niches. Similarly, among the 1,011 sequenced strains, $37 \%$ of isolates from wild environments such as trees, insects, water or soil were heterozygous and their heterozygosity level was comparable to that of domesticated or human-related strains (Figure 3). Although these could represent instances of feral isolates, with domesticated strains re-invading natural environments, it is difficult to conclusively discriminate feralization from the opposing scenario of wild strains entering the human made environments and being domesticated. In conclusion, wild populations of $S$. cerevisiae are unexpectedly heterozygous, and this feature might have been further enriched in human-related environments to give rise to the extant domesticated heterozygotes [Liti et al. 2009]. In this context, how heterozygosity is generated and maintained in the population remains a mystery.

\section{A surprising prevalence of polyploidy}

The canonical $S$. cerevisiae life cycle predicts a strict alternation between haploid and diploid phases. However, a large proportion of strains isolated from diverse environments including 
the "Evolution Canyon", and industrial processes such as brewing and baking, are triploids or tetraploids [Ezov et al. 2006; Al Safadi, Weiss-Gayet, Briolay, and Aigle 2010; Gallone et al. 2016]. In addition, about $13 \%$ of the 1,011 S. cerevisiae isolates are also polyploids and the proportion of polyploids is comparable between domesticated and wild strains [Peter et al. 2018] (Figure 4).

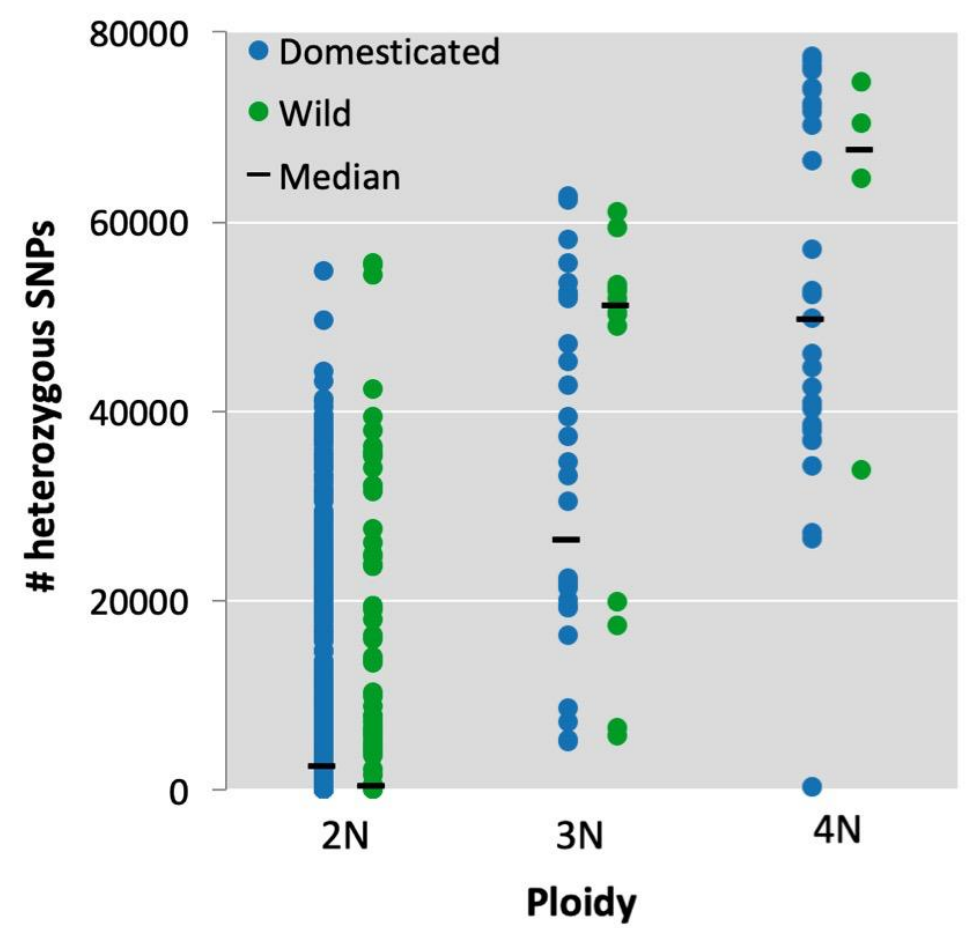

Figure 4: Number of heterozygous SNPs as a function of ploidy in the 1,011 strains depending on their ecological niche "Domesticated" or "Wild".

Two main types of polyploidization can lead to tetraploids. Autopolyploidy yields genome duplication within one species while allopolyploidy implies the merging of two genomes in a single nucleus following interspecific hybridization [Alix, Gérard, Schwarzacher, and HeslopHarrison 2017]. This review specifically covers intra-species events, we will therefore only focus on autopolyploids.

Autopolyploids can be formed by distinct molecular mechanisms. Whole genome duplication (WGD) by endoreduplication or cytokinesis failure can produce tetraploids from diploid cells. Alternatively, homozygotization of the MAT locus by unprogrammed mating type switching in a diploid cell, followed by "mating" with a haploid cell can generate a triploid [Storchova 2014]. Tetraploid could also be generated through mating between 2 diploid cells having acquired opposite mating types by unprogrammed mating type switching (Figure 5). The quantitative impact of unprogrammed mating type switching might have been underappreciated at the population level. We found that 159 diploid strains among 698 diploids from the 1,011 collection (23\%) are unable to sporulate. While some of them might have mutations disabling sporulation [Chiara et al. 2020], others may have undergone 
unprogrammed mating type switching. An elegant study from the Kupiec group recently showed the relatively high rate of both unprogrammed mating type switching and endoreduplication in a heterothallic haploid laboratory strain background [Harari, Ram, Rappoport, Hadany, and Kupiec 2018]. Lacefield and collaborators described another possible mechanism leading to polyploidy [Tsuchiya, Yang, and Lacefield 2014]. They showed that establishing a proper meiotic commitment through the Ndt80 positive feedback loop was important in maintaining ploidy. A reduction of Ndt80 during meiosis resulted in inappropriately uncommitted cells that can return to mitosis at stages beyond prometaphase I. The failure to commit to meiosis resulted in the formation of multi-nucleate polyploid cells which replicate their DNA prior to the mitotic division, leading to mother cells with 2-3 nuclei and a $4 \mathrm{~N}-6 \mathrm{~N}$ DNA content.

The large number of polyploids in the population suggests that the mechanisms of polyploidization could be more frequent than classically envisioned and/or that the polyploid state could be beneficial under specific environments [Todd, Forche, and Selmecki 2017]. The latter was shown for tetraploids that maintain stable genomes during long-term growth at $23^{\circ} \mathrm{C}$ [Selmecki et al. 2015; Lu, Swamy, and Leu 2016]. Several studies showed that polyploids can be highly unstable and undergo rapid ploidy reduction during non-meiotic experimental evolution [Selmecki et al. 2015; Gerstein, Chun, Grant, and Otto 2006; Gerstein, McBride, and Otto 2008]. However, these experiments were performed with homozygous polyploids and the propensity of heterozygous polyploid to regress towards diploidy during vegetative growth is unknown. Either way, the prevalence of autopolyploids in S. cerevisiae escapes the simple alternation between haploid and diploid phases suggesting that polyploidization in the yeast life cycle and its contribution to the genetic makeup of the species is underappreciated.

\section{Heterozygosity level positively correlates with ploidy}

The 1,011 genome sequences revealed a strong association between heterozygosity and polyploidy [Peter et al. 2018]. All triploid and tetraploid isolates, with the exception of one domesticated tetraploid, are highly heterozygous and the median heterozygosity level positively correlates with ploidy (Figure 4). Additionally, Figure 4 shows that the repartition of heterozygosity and polyploidy is comparable between domesticated and wild strains among the 1,011 sequenced isolates. We also observed that wild polyploids show a median heterozygosity level higher than domesticated strains which is not consistent with the general idea that domestication would promote the formation of heterozygotes.

The relationship between polyploidy formation and heterozygosity is unknown but their strong association suggests that polyploids could result from mating between diverged lineages. This could be promoted by the formation of mating diploids after unprogrammed 
homozygotization of their mating type loci. Although WGD (endoreduplication or cytokinesis failure) appears less likely because it cannot directly account for the presence of triploids, it can formally produce heterozygous tetraploids when the original diploid is heterozygous. Remarkably, spontaneous WGD is a way to restore meiotic fertility in non-sporulating interspecific hybrids [Greig, Borts, Louis, and Travisano 2002; Charron, Marsit, Hénault, Martin, and Landry 2019]. Another possibility to explain the predominance of heterozygous polyploids is that both homo- and heterozygous polyploids would be equally formed but only heterozygous polyploids would persist because of higher chromosome stability or higher fitness due to heterosis [Comai 2005]. The allele frequencies (AF) of heterozygous sites can provide insights into the formation of polyploids. For example, abundance of heterozygous sites at $0.5 \mathrm{AF}$ could underlie mating between two homozygous diploids from different genetic backgrounds. Mixture of heterozygous sites with $0.25,0.5$ and $0.75 \mathrm{AF}$ would suggest more complex scenario. In addition, the analysis of the haplotype structures can be informative to trace the founder populations of the polyploids [Fay, Liu, Ong, Dunham, Cromie, Jeffery, Ludlow, and Dudley 2019]. Overall, the relative proportions of heterozygous sites and their allele frequencies in polyploids are important to infer the possible scenario leading to polyploidy.

Why cells from diverged lineages would be more prone to 'mate' than cells from the same genetic background remains an open question. However, an enlightening case of dioecy (separation of sexes) exemplifies several hypotheses that are discussed in this review. $S$. cerevisiae is formally a hermaphrodite since one cell can give rise to gametes able to mate among them and regenerate a parental organism. The group of Michel Aigle reported a case of near-dioecy in a $S$. cerevisiae population isolated from pearl millet beer, where the diploid meiotic progenies of tetraploid parental isolates comprise both non-mating MATa/ $\alpha$ diploids, as expected, but also MATa/a diploids able to mate with either MATa or MATa cells but not both [Al Safadi, Weiss-Gayet, Briolay, and Aigle 2010]. This latter feature is unique so far in the budding yeast field and illustrates a case of near-dioecy where the two sexes of the population are separated, which eventually imposes outcrossing and therefore would strongly promote heterozygosity among this tetraploid population. How general is dioecy in the population remains unexplored.

\section{Open questions on the life cycle in S. cerevisiae}

Overall, the presence of large numbers of highly heterozygous and polyploid isolates in both wild and domesticated yeast populations show that either they originate at unexpectedly high rates and/or they persist longer than commonly assumed from our vision of the canonical life cycle. We hypothesize that in addition to outcrossing, alternative and underappreciated routes could shape the yeast natural life cycle to generate and maintain heterozygosity 11 
through polyploidization (Figure 5). These routes can be purely random such as unprogrammed mating type switching and errors during mitosis, meiosis or mating. Other might routes be controlled, which would enforce the system, and include heterothallism, reduced spore formation and viability, and dioecy. Only by assessing the frequencies and genetic control these additional routes at the population level, we will be able to test our hypothesis, which might have implications beyond the yeast life-cycle.

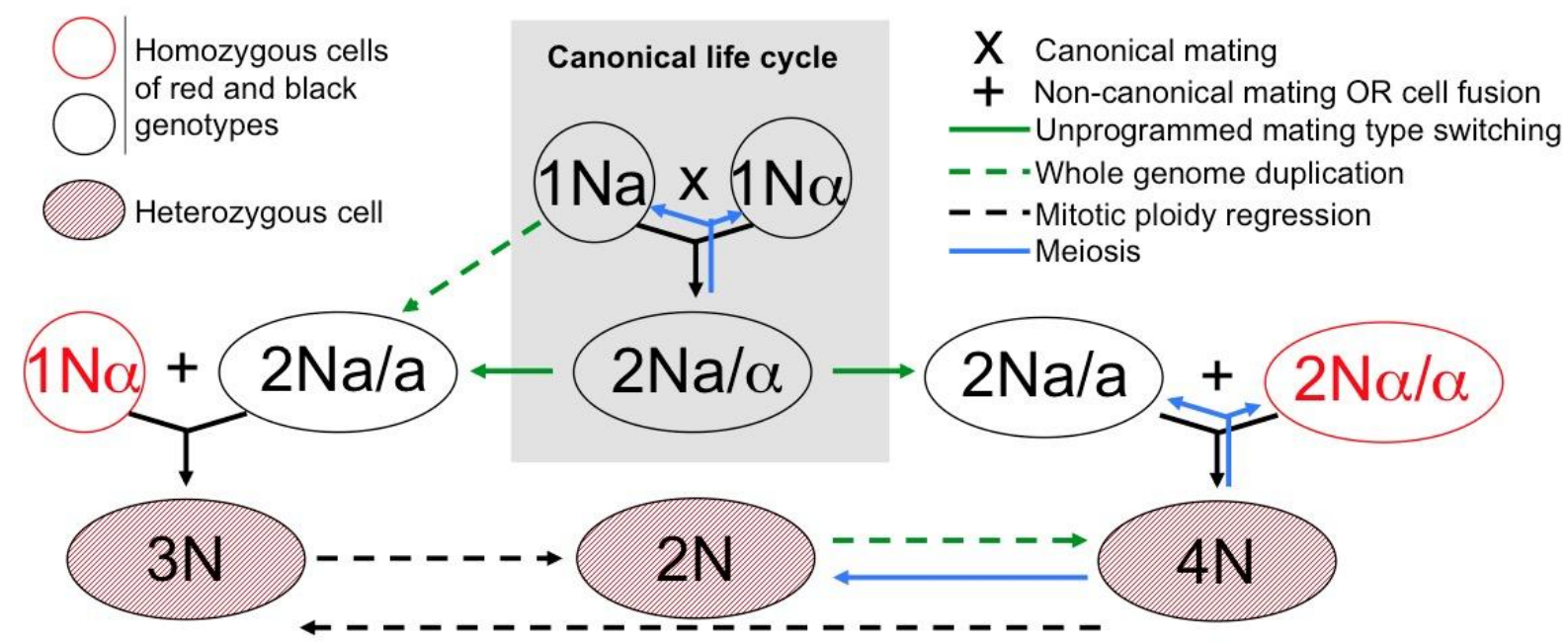

Figure 5: Possible adaptations to the canonical life cycle. Mitotic ploidy regression likely involves a progressive loss of chromosomes involving intermediate aneuploidy states. 


\section{References}

Al Safadi R, Weiss-Gayet M, Briolay J, Aigle M. 2010. A polyploid population of Saccharomyces cerevisiae with separate sexes (dioecy). FEMS Yeast Res., 10: 757-768.

Alix K, Gérard PR, Schwarzacher T, Heslop-Harrison JSP. 2017. Polyploidy and interspecific hybridization: partners for adaptation, speciation and evolution in plants. Ann. Bot., 120: 183194.

Charron G, Marsit S, Hénault M, Martin H, Landry CR. 2019. Spontaneous whole-genome duplication restores fertility in interspecific hybrids. Nat. Commun., 10: 1-10.

Chiara MD, Barré B, Persson K, Chioma AO, Irizar A, Schacherer J, Warringer J, Liti G. 2020. Domestication reprogrammed the budding yeast life cycle. bioRxiv, 2020.02.08.939314.

Comai L. 2005. The advantages and disadvantages of being polyploid. Nat. Rev. Genet., 6: 836-846.

Duan S-F, Han P-J, Wang Q-M, Liu W-Q, Shi J-Y, Li K, Zhang X-L, Bai F-Y. 2018. The origin and adaptive evolution of domesticated populations of yeast from Far East Asia. Nat. Commun., 9: 2690.

Dutta A, Lin G, Pankajam AV, Chakraborty P, Bhat N, Steinmetz LM, Nishant KT. 2017. Genome Dynamics of Hybrid Saccharomyces cerevisiae During Vegetative and Meiotic Divisions. G3 Genes Genomes Genet., 7: 3669-3679.

Ezov TK, Boger-Nadjar E, Frenkel Z, Katsperovski I, Kemeny S, Nevo E, Korol A, Kashi Y. 2006. Molecular-Genetic Biodiversity in a Natural Population of the Yeast Saccharomyces cerevisiae From "Evolution Canyon": Microsatellite Polymorphism, Ploidy and Controversial Sexual Status. Genetics, 174: 1455-1468.

Ezov TK, Chang S-L, Frenkel Z, Segrè AV, Bahalul M, Murray AW, Leu J-Y, Korol A, Kashi Y. 2010. Heterothallism in Saccharomyces cerevisiae isolates from nature: effect of $\mathrm{HO}$ locus on the mode of reproduction. Mol. Ecol., 19: 121-131.

Fay JC, Liu P, Ong GT, Dunham MJ, Cromie GA, Jeffery EW, Ludlow CL, Dudley AM. 2019. A polyploid admixed origin of beer yeasts derived from European and Asian wine populations. PLOS Biol., 17: e3000147.

Gallone B et al. 2016. Domestication and Divergence of Saccharomyces cerevisiae Beer Yeasts. Cell, 166: 1397-1410.e16.

Gerke J, Lorenz K, Cohen B. 2009. Genetic Interactions Between Transcription Factors Cause Natural Variation in Yeast. Science, 323: 498-501.

Gerstein AC, Chun H-JE, Grant A, Otto SP. 2006. Genomic convergence toward diploidy in Saccharomyces cerevisiae. PLoS Genet., 2: e145.

Gerstein AC, McBride RM, Otto SP. 2008. Ploidy reduction in Saccharomyces cerevisiae. 
Biol. Lett., 4: 91-94.

Goddard MR, Anfang N, Tang R, Gardner RC, Jun C. 2010. A distinct population of Saccharomyces cerevisiae in New Zealand: evidence for local dispersal by insects and human-aided global dispersal in oak barrels. Environ. Microbiol., 12: 63-73.

Greig D, Borts RH, Louis EJ, Travisano M. 2002. Epistasis and hybrid sterility in Saccharomyces. Proc. Biol. Sci., 269: 1167-1171.

Haber JE. 1998. Mating-type gene switching in Saccharomyces cerevisiae. Annu. Rev. Genet., 32: 561-599.

Haber JE. 2012. Mating-type genes and MAT switching in Saccharomyces cerevisiae. Genetics, 191: 33-64.

Haber JE, Savage WT, Raposa SM, Weiffenbach B, Rowe LB. 1980. Mutations preventing transpositions of yeast mating type alleles. Proc. Natl. Acad. Sci. U. S. A., 77: 2824-2828.

Harari Y, Ram Y, Rappoport N, Hadany L, Kupiec M. 2018. Spontaneous Changes in Ploidy Are Common in Yeast. Curr. Biol. CB, 28: 825-835.e4.

Kelly AC, Shewmaker FP, Kryndushkin D, Wickner RB. 2012. Sex, prions, and plasmids in yeast. Proc. Natl. Acad. Sci. U. S. A., 109: E2683-2690.

Knop M. 2006. Evolution of the hemiascomycete yeasts: on life styles and the importance of inbreeding. BioEssays, 28: 696-708.

Liti G et al. 2009. Population genomics of domestic and wild yeasts. Nature, 458: 337-341.

Liti G. 2015. The fascinating and secret wild life of the budding yeast S. cerevisiae. eLife, 4: e05835.

Llorente B, Smith CE, Symington LS. 2008. Break-induced replication: what is it and what is it for? Cell Cycle Georget. Tex, 7: 859-864.

Lu Y-J, Swamy KBS, Leu J-Y. 2016. Experimental Evolution Reveals Interplay between Sch9 and Polyploid Stability in Yeast. PLoS Genet., 12: e1006409.

Magwene PM. 2014. Revisiting Mortimer's Genome Renewal Hypothesis: heterozygosity, homothallism, and the potential for adaptation in yeast. Adv. Exp. Med. Biol., 781: 37-48.

McClure AW, Jacobs KC, Zyla TR, Lew DJ. 2018. Mating in wild yeast: delayed interest in sex after spore germination. Mol. Biol. Cell, 29: 3119-3127.

Meiron H, Nahon E, Raveh D. 1995. Identification of the heterothallic mutation in HOendonuclease of S. cerevisiae using HO/ho chimeric genes. Curr. Genet., 28: 367-373.

Mortimer RK, Romano P, Suzzi G, Polsinelli M. 1994. Genome renewal: A new phenomenon revealed from a genetic study of 43 strains of Saccharomyces cerevisiae derived from natural fermentation of grape musts. Yeast, 10: 1543-1552.

Murphy HA, Zeyl CW. 2010. Yeast Sex: Surprisingly High Rates of Outcrossing between Asci. PLOS ONE, 5: e10461.

Nishant KT et al. 2010. The Baker's Yeast Diploid Genome Is Remarkably Stable in 14 
Vegetative Growth and Meiosis. PLOS Genet., 6: e1001109.

Peter $J$ et al. 2018. Genome evolution across 1,011 Saccharomyces cerevisiae isolates. Nature, 556: 339.

Ray BL, White Cl, Haber JE. 1991. Heteroduplex formation and mismatch repair of the "stuck" mutation during mating-type switching in Saccharomyces cerevisiae. Mol. Cell. Biol., 11: 5372-5380.

Reuter M, Bell G, Greig D. 2007. Increased outbreeding in yeast in response to dispersal by an insect vector. Curr. Biol., 17: R81-R83.

Ruderfer DM, Pratt SC, Seidel HS, Kruglyak L. 2006. Population genomic analysis of outcrossing and recombination in yeast. Nat. Genet., 38: 1077-1081.

Selmecki AM et al. 2015. Polyploidy can drive rapid adaptation in yeast. Nature, 519: 349352.

Storchova Z. 2014. Ploidy changes and genome stability in yeast. Yeast, 31: 421-430.

Tattini L, Tellini N, Mozzachiodi S, D’Angiolo M, Loeillet S, Nicolas A, Liti G. 2019. Accurate Tracking of the Mutational Landscape of Diploid Hybrid Genomes. Mol. Biol. Evol., 36: 28612877.

Taxis C, Keller P, Kavagiou Z, Jensen LJ, Colombelli J, Bork P, Stelzer EHK, Knop M. 2005. Spore number control and breeding in Saccharomyces cerevisiae: a key role for a selforganizing system. J. Cell Biol., 171: 627-640.

Todd RT, Forche A, Selmecki A. 2017. Ploidy Variation in Fungi - Polyploidy, Aneuploidy, and Genome Evolution. Microbiol. Spectr., 5. https://www.ncbi.nlm.nih.gov/pmc/articles/PMC5656283/ (Accessed January 24, 2020).

Tsai IJ, Bensasson D, Burt A, Koufopanou V. 2008. Population genomics of the wild yeast Saccharomyces paradoxus: Quantifying the life cycle. Proc. Natl. Acad. Sci. U. S. A., 105: 4957-4962.

Tsuchiya D, Yang Y, Lacefield S. 2014. Positive feedback of NDT80 expression ensures irreversible meiotic commitment in budding yeast. PLoS Genet., 10: e1004398. 


\section{Legend to figures}

Figure 1: Canonical mating strategies

Figure 2: $H O$ diversity across the 1,011 genomes. The circle diameter is proportional to the number of strains. The red circle comprise 93 strains and represents the inactive form of $\mathrm{HO}$ present in most laboratory strains. The green circle comprises 307 strains carrying the active $\mathrm{HO}$ sequence. The blue circles contain strains for which $\mathrm{HO}$ functionality is unknown. However, all truncated forms of $\mathrm{HO}$ are likely heterothallic.

Figure 3: Distribution of the number of heterozygous SNPs in the 794 strains sequenced in their original ploidy and heterozygosity levels (Peter et al., Nature 2018) as a function of their ecological niche. "Domesticated" means strains associated with human activity (fermentation, bakery), "Human" means strains isolated from human body or human-related environment such as cultivated fruits and flowers, "Wild" means strains isolated from natural environments

Figure 4: Number of heterozygous SNPs as a function of ploidy in the 1,011 strains depending on their ecological niche "Domesticated" or "Wild".

Figure 5: Possible adaptations to the canonical life cycle. Mitotic ploidy regression likely involves a progressive loss of chromosomes involving intermediate aneuploidy states. 
Trashed

Saccharomyces cerevisiae has served as a model for nearly a century to understand the principles of the eukaryotic life cycle.

Both inbreeding and haploselfing promote a rapid loss of heterozygosity and therefore most natural isolates are expected to be homozygous diploids. Contrary to this expectation,

In this context, how heterozygosity and polyploidy are generated and maintained in the population remains a mystery. 